BULLETIN Bulletin hispanique

HISPANIQUE Université Michel de Montaigne Bordeaux

115-1 | 2013

Poésie et société en Espagne : 1650-1750

\title{
La poésie scientifique de Bernardino de Rebolledo à la lumière du gassendisme
}

\section{Frédéric Prot}

\section{(2) OpenEdition}

1 Journals

\section{Edición electrónica}

URL: http://journals.openedition.org/bulletinhispanique/2308

DOI: 10.4000/bulletinhispanique.2308

ISSN: 1775-3821

\section{Editor}

Presses universitaires de Bordeaux

\section{Edición impresa}

Fecha de publicación: 1 junio 2013

Paginación: 13-26

ISBN: 978-2-86781-898-1

ISSN: 0007-4640

\section{Referencia electrónica}

Frédéric Prot, «La poésie scientifique de Bernardino de Rebolledo à la lumière du gassendisme », Bulletin hispanique [En línea], 115-1 | 2013, Publicado el 01 junio 2016, consultado el 06 mayo 2019.

URL : http://journals.openedition.org/bulletinhispanique/2308; DOI : 10.4000/bulletinhispanique.2308 


\title{
La poésie scientifique de Bernardino de Rebolledo à la lumière du gassendisme
}

\author{
FRÉdÉRIC PROT \\ Université Michel de Montaigne Bordeaux 3
}

Le gassendisme offre une nouvelle lecture de la poésie scientifique de Rebolledo et de sa lettre de réhabilitation d'Épicure. D'une position de rejet de l'héliocentrisme copernicogaliléen, Rebolledo évoluera vers une opinion favorable au système mixte de Tycho Brahé. Un scepticisme chrétien oriente le sens de sa contemplation du monde, proche de celle de Gassendi, dont les auvres étaient familières à Rebolledo, comme l'atteste la mention de la théorie des espaces imaginaires.

Mots clés : Rebolledo, Gassendi, physique, astronomie, scepticisme, epicure.

El gasendismo ofrece una nueva lectura de la poesía cientifica de Rebolledo y de su carta de rehabilitación de Epicuro. Desde una postura de rechazo del heliocentrismo copernicanogalileano, Rebolledo evolucionará hacia una opinión favorable del sistema mixto de Tycho Brahé. Un escepticismo cristiano orienta el sentido de su contemplación del mundo, que se asemejará a la de Gassendi cuyas obras eran familiares para Rebolledo, como lo atestiguan en particular la mención de la teoría de los espacios imaginarios.

Palabras claves: Rebolledo, Gassendi, Física, Astronomía, Escepticismo, Epicuro.

"Gassendism» offers a new reading of Rebolledo's scientific poetry as of his letter on rehabilitating Epicurus, written in the light of the De Vita et "Moribus Epicuri». From a rejection of the Copernico-Galilean heliocentric theory, the Ambassador of Philip IV at the Danish Court will evolve towards an opinion favourable to the mixed system of Tycho Brahe. A Christian scepticism runs through his contemplation of the world, closer to Gassendi's, whose Physics and philosophical and moral work were well known by Rebolledo, as ascertains, in particular, the mention of the "imaginary spaces» theory, connected to Epicurean space understood as real entity.

Keywords: Rebolledo, Gassendi, Physics, Astronomy, Scepticism, Epicurus. 
B ernardino de Rebolledo (1597-1676) et Pierre Gassendi (1592-1655) B participent tous deux de l'humanisme tardif. Leur pensée ne se sépare pas de l'ensemble de l'histoire de la philosophie dont ils perçoivent la continuité par-delà la disparité des opinions et des systèmes ${ }^{1}$. Rebelles au dogmatisme scolastique, ils prônent tous deux la liberté féconde du scepticisme, sans s'égarer dans l'indifférence ataraxique de la volonté qui ferait de cette même liberté une liberté vide.

Une longue mission au Danemark (1648-1661) - pays protestant, patrie de l'astronome Tycho Brahé - en qualité d'ambassadeur de Philippe IV, l'éclectisme de ses lectures et les conversations qu'il entretint avec Caramuel ${ }^{2}$ et d'autres libres esprits forgent la singularité de l'œuvre de Rebolledo, dont on propose ici une lecture à la lumière du gassendisme. Quoique relevant bien plus de la forme du fragment que du discours conduit de ses attendus à ses corollaires, sa poésie scientifique et certaines compositions en prose révèlent de profondes parentés - parfois explicitement revendiquées - avec la philosophie de Gassendi. Ainsi sa lettre de réhabilitation d'Épicure ${ }^{3}$ révèle-t-elle l'empreinte sous-jacente du De Vita et Moribus Epicuri (1647), dont le modèle lui permet de dresser la carte des confluences entre épicurisme, stoïcisme et christianisme. De même, la vraisemblance de l'héliocentrisme incite Rebolledo à se rallier, comme Gassendi, au système de compromis de Tycho Brahé. Comme l'auteur des Exercitationes et du Syntagma, Rebolledo se défie des incertaines catégories de l'être forgées par les philosophes, tel Aristote, afin de saisir l'essence du réel et préfère la vraisemblance à la vérité, la probabilité à la certitude. Le traité De la Sagesse (1601) de Pierre Charron est une lecture commune aux deux hommes.

Après avoir exposé les termes du scepticisme chrétien de Rebolledo et le sens de la contemplation du monde, on examinera le cheminement intellectuel qui le mène du rejet de l'héliocentrisme à une position plus circonspecte. La mention de la théorie gassendiste des espaces imaginaires, qu'il relie à l'espace épicurien entendu comme être réel, révèle cet effort discret de recomposition de la continuité philosophique et scientifique dans le temps.

1. Cf. Olivier Bloch, La philosophie de Gassendi. Nominalisme, matérialisme et métaphysique, La Haye, Martinus Nijhoff, 1971, p.44.

2. En 1644, Rebolledo avait accueilli Juan Caramuel entre les murs de sa forteresse de Frankenthal. Celui-ci évoque à plusieurs reprises leurs conversations amicales et érudites (Pandoxion physico-eticum. Campaniae, 1668, p. 158 ; Mathesis Biceps, Campaniae, 1670, p. 469). Les travaux de Gassendi, dont Caramuel était le correspondant, furent sans doute discutés alors.

3. "Este papel escribió el autor al Señor barón Don Juan de Goes ", Selva militar y política, Madrid, Antonio de Sancha, 1661, p. 477-495. 
$Y$ de las calidades, de la materia y forma, / De elementos, o puros o alterados, / Mixtos inanimados, / Plantas, árboles, frutos, y simientes, / Aves, peces, reptiles, animales, / Hago especulaciones diferentes, / Y tal vez experiencia, / Sin reducirla inutilmente a ciencia. I Ni examinar con pérdida de tiempo / Si de las continuadas cantidades / La división en partes I ha de ser infinita / Como quiere el sutil Estagirita, I o cual Cenón en puntos, / De límite infalible, / cada uno en si mismo indivisible; / Ni si podrán juntar sus unidades / Las que quiere Epicuro inanidades ${ }^{4}$.

$\mathrm{Ni}$ résiliation de la physique d'Aristote ni affiliation à celle d'Épicure : Rebolledo met plutôt deux systèmes dos à dos, hostile à la réduction de la Création à une physique soit trop artificieuse dans ses constructions de la raison pure, soit trop spéculative dans son intellection du réel. L'armature conceptuelle aristotélicienne (matière, forme, mixtes), sa méthodologie et sa théorie de la division infinie de la matière lui inspirent perplexité autant que celle épicurienne de l'atome, principe corporel insécable en quoi se réduit tout ce qui est. Un tel scepticisme est remarquable en un temps où la physique scolastique impose sa domination. Ces vers expriment peut-être d'abord une polémique d'ordre épistémologique, destinée à rabattre les prétentions de la science à (re)formuler et forcer le réel selon les notions et concepts de la raison. Une défiance qui oriente toute l'œuvre de Gassendi' ${ }^{5}$. Tous deux défendent une position agnostique sur le terrain du savoir, en refusant aussi bien à l'atomisme antique qu'à la physique aristotélicienne la valeur d'une représentation de la nature qui en révélerait les ressorts.

Rebolledo semble aspirer à l'ataraxie lucrétienne du Suave mari magno, à l'abri des tempêtes des fausses certitudes. Sa raison, désemparée par sa propre indécidabilité face aux querelles philosophiques, trouve la paix dans l'échappée du regard simplement posé sur la Création qui lui communique son mystère. Le monde des essences est impénétrable. Rebolledo professe une docte ignorance, sans démunir la science de sa capacité à rendre intelligible le réel :

Deseando que el alma / De agitación descanse molesta, / Y en nunca de ellas perturbada calma / Con vuelo aspire menos imperfecto / Encubrándose de uno en otro objeto / A la contemplación del soberano, / Último fin de todo afecto humano: / Cuyo menos o más conocimiento / De la felicidad es argumento. / Esta sola doctrina / Será de tus pesares / Bastante medicina, / Si el ánimo aplicares / En que ocultas existen las esencias / Orígenes formales de las ciencias, / A los de sus primeros rudimentos / Leves, pero eficaces documentos ${ }^{6}$.

Chez Rebolledo comme chez Gassendi, l'indifférence de la volonté du sceptique chrétien semble ainsi moins libertas que libentia, "bon vouloir " : "Le plus haut degré de liberté peut être tenu pour celui qui, reposant sur

4. Rebolledo, Selvas dánicas, «Segunda Selva », in Ocios, Ámberes, Oficina Plantiniana, 1660, p. 493-494.

5. Cf. Bloch, op. cit., p. 312. Gassendi publie en 1624 ses Exercitationes paradoxicae adversus Aristoteleos, où il remet en cause les dogmes et les enseignements scolastiques.

6. Selvas dánicas, p. 496-497. 
la connaissance parfaite du souverain bien par l'entendement, consiste pour la volonté à adhérer nécessairement à ce souverain bien, à aimer Dieu d'un consentement à la fois nécessaire et spontané ${ }^{7}$. "Rebolledo trouve la sérénité dans l'acceptation pure de son être-au-monde :

Ten [go] por doctrina más constante / Lo indivisible del presente instante. / Espero que vendrá la primavera / De pámpanos y flores coronada, / De mieses el verano, / El otoño de fruta sazonada ; / El invierno de hielo y niebe cano, / Y con leve mudanza / Rara vez me ha engañado la Esperanza. I Investigo las causas / De esta infalible variedad de efectos, I Los impulsos violentos / De los feroces y contrarios vientos; / Del vapor, que de tierra y agua sube / A convertirse en nube ${ }^{8}$.

Le scepticisme se transmue en contemplation chrétienne de l'œuvre de la Nature, de la mécanique invariable des saisons et des phénomènes climatiques. Lindifférence aux querelles philosophiques n'aboutit pas à la liberté du néant, mais se résout dans la jouissance admirative de ce spectacle. L'homme ne peut atteindre aux essences et vérités intérieures des choses. Inaccessible aux causes, il ne peut qu'observer la Création dans ses effets et ses apparences car on ne connaît de la substance que ses attributs extérieurs et ses propriétés utiles. La condition humaine est une condition de spectateur. Dieu donne à voir le jeu de la machine du Monde sans en dévoiler la fabrique. Gassendi invite de même à regarder les choses purement et simplement, car tout se réduit à voir: de toutes choses, on n'a qu'une conception imparfaite et déformée par l'abstraction de la raison. La portée de la spéculation mais aussi de l'activité expérimentale dans les sciences de la nature est donc limitée 9 : "Bien que, de cette science que j'ai coutume d'appeler science des "phénomènes" ou science "Historique", je puisse saisir quelque bribe, en revanche de cette autre science illustre, par quoi l'on connaîtrait les natures intimes et les propriétés des choses, je ne me fais pas crédit du moindre atome. C'est une science que j'accorde toute entière à Dieu, parce que, ouvrier de la Nature, lui seul peut dominer du regard le plan de son ouvrage ${ }^{10}$.»

Rebolledo, comme Gassendi ${ }^{11}$, marque sa perplexité face à l'idée de progrès du savoir. Sa quantité augmente, mais il est abusif de parler d'essor exponentiel de l'esprit humain - comme on le fera au XVIII siècle. La science non seulement peine à éclairer la Création mais, par une ironie du sort, la multiplicité des systèmes se disputant la vérité tend à ramener celle-ci à ses premiers balbutiements :

Subo tal vez al monte con los contemplativos y ya que no al Cielo por la escala de San Juan Climaco, o moradas de Santa Teresa, a medir sus distancias con Tolomeo y Clavio, si bien temo embarazarme en una ciencia que siendo la primera que se empezó a especular

7. Bloch, op. cit., p. 64.

8. Selvas dánicas, p. 494.

9. Cf. Bloch, op. cit., p. 71 sqq.

10. Gassendi, lettre à Louis de Valois du 28 juin 1641, in Bloch, op. cit., p. 83.

11. Cf. Bloch, op. cit., p. 73 sqq. 
después de tantos siglos de observaciones no acaba de asegurar sus principios, y debatiéndolos de nuevo de puro decrepita parece que se vuelve a la edad de los niños ${ }^{12}$.

Les aspirations scientifiques de Rebolledo comme de Gassendi relèvent d'une modernité mitigée, dont le profil est celui d'une " philosophie de compromis circonspecte, éclectique et relativiste $»^{13}$. Leur scepticisme et leur mise en cause de la perfection du savoir ne s'inscrivent pas pour autant dans le sillage du pyrrhonisme. Ni l'incertitude irrémédiable de celui-ci ni le dogmatisme des vérités sûres d'elles-mêmes ne pèsent sur leur intelligence du monde. Le salut est un repli sur la connaissance vraisemblable et conjecturale. Cette inclination au probabilisme explique les vacillations de Rebolledo face à l'héliocentrisme. La probabilité est une moindre certitude mais c'est s'accorder à la qualité imparfaite de la connaissance ${ }^{14}$ qui ne peut saisir que les apparences et les phénomènes et non pas l'être en soi. Tel est l'esprit du Syntagma ${ }^{15}$. Du cosmos à portée de lunettes astronomiques, à la nature terrestre à portée de regard, l'être résiste :

Del sol, la luna y los demás planetas / Observo alguna vez los movimientos, / Sin dejarme vencer al de la tierra, / [...] / Y de este ángulo breve / Con lineas imperfectas / Por meridianos corro y paralelos / El ámbito espacioso de los cielos, / Sin perdonar constelación ni estrella / Desde la menos clara a la más bella ; I Y con mayor estudio me prevengo / A conocer lo que más cerca tengo, / Y más dificil atención me cuesta / El medir mis pasiones, / Que del sur a los rígidos triones ${ }^{16}$.

Homme, nature et cosmos : le fond des choses, les structures intérieures du monde, les plis de l'âme demeurent voilés ${ }^{17}$. Des limites s'imposent aussi bien à la modalité de la connaissance qu’à son contenu et son étendue.

La poésie scientifique de Rebolledo est trop fragmentaire pour que l'on puisse en inférer l'expression de ce nominalisme ontologique qui commande l'œuvre de Gassendi ${ }^{18}$ et repose sur l'idée que la connaissance ne passe pas par la considération de principes abstraits de la pensée en prise sur l'être. Il n'y a d'existence que foncièrement singulière. Il n'est d'autres universalités que celle des concepts et des noms. Toute proposition vraie porte sur un sujet et un phénomène existant dans le présent et non sur une essence intemporelle.

Rebolledo s'en remet à la coïncidence harmonieuse de son existence avec celle de la Nature providentielle ${ }^{19}$. "Ainsi la condition de la connaissance humaine, qui, comme connaissance, doit nécessairement s'appuyer sur une vision, mais qui, comme humaine, ne peut s'appuyer sur une vision intellectuelle et ne peut

12. Rebolledo, "Carta del autor a Don Ramiro de Quiñones », Ocios, p. 192.

13. Bloch, op. cit., p. 76.

14. Cf. Bloch, op. cit., p. 92.

15. Cf. Bloch, op. cit., p. 96.

16. "Segunda Selva », p. 495-496.

17. Cf. Bloch, op. cit., p. 98.

18. Cf. Bloch, op. cit., p. 111 sqq.

19. "Segunda Selva », p. 493-494. 
partir que d'une vision sensible, se trouve-t-elle dès lors référée au rapport de dépendance métaphysique de l'homme à Dieu, et c'est à ce rapport que renvoient le statut de notre intellection, l'impossibilité pour une idée humaine de représenter l'infini ${ }^{20}$. "S'il est hasardeux de reconnaître dans la poésie de Rebolledo l'attitude empiriste et naturaliste qui fonde la critique de Gassendi contre tout formalisme logique, on peut cependant reconnaittre une parenté jusque dans le finalisme.

Dans une page du Syntagma ${ }^{21}$, Gassendi déclare que, faute de pouvoir accéder à la formule du monde, c'est un grand bonheur que de jouir du Théâtre de la nature, de son harmonie et de sa finalitée 22 . La contemplation d'un art et d'une sagesse qui ne sont pas les nôtres ne ressortit pas pour autant à une passivité de la vision admirative. La science contemplative qu'est la physique ${ }^{23}$, investie d'une signification théologique ${ }^{24}$, est possible dans la mesure où Dieu, dans sa bonté, la légitime. La physique gassendiste forme une "invitation à contempler dans la nature le fonctionnement d'un automatisme bien construit et bien réglé par un artisan qui seul en connaît les ressorts intimes ${ }^{25}$. Et Rebolledo de contempler le cours des saisons et les variations du climat.

Rebolledo est féru de théologie, de lectures ${ }^{26}$ et d'exégèses bibliques. Ses fonctions d'ambassadeur à la cour danoise, en pays protestant, le vouent à un isolement qui exalte l'éclat de sa foi inquiète de catholique et favorise aussi un esprit de libre curiosité. Les historiens ont établi le rôle important qu'il joua dans les plans secrets de conversion de Christine de Suède au catholicisme ${ }^{27}$. La Constancia victoriosa (1655), qui lui est dédiée, est une traduction du livre de Job, suivie d'une, seconde, personnelle, des «Lamentations de Jérémie ${ }^{28}$. En 1659, voit le jour une traduction complète des Psaumes: la Selva sagrada. Enfin, en 1660, Rebolledo offre une paraphrase de la Passion du Christ, inspirée de l'Evangile de saint Jean : l'Idilio sacro. A l'adresse d'un docteur de

20. Bloch, op. cit., p. 460.

21. Cf. Syntagma, in Opera omnia, Lyon, L. Anisson, 1658, I, 79b-80a.

22. La finalité est « la voie royale de la théologie gassendiste " (Bloch, op. cit., p. 437).

23. Cf. Bloch, op. cit., p. 456.

24. Cf. Gassendi, Proemium de la Physique du Syntagma. Cf. Bloch, op. cit., p. 434.

25. Bloch, op. cit., p. 436-437.

26. Dans une lettre d'avril 1651, Rebolledo exprime l'inquiète passion avec laquelle il aime plonger le soir son esprit dans les Saintes Écritures ("Carta a Don Ramiro de Quiñones », Ocios, p. 191-192).

27. Cf. Oskar Garstein, Rome and the Counter-reformation in Scandinavia, Leiden, E. J. Brill, 1992 ; Sven Ingemar Olofsson, Drottning Christinas tronavsägelse och trosföränding, Uppsala, 1953, p. 164-165, p. 183, p. 193-194 ; Emil Gigas, Grev Bernardino de Rebolledo, spansk gesandt i Kjøbenhaven. 1648-1659, Kjøbenhavn, I.H. Schubothes Boghandel, 1883.

28. Vásquez Lopera soutient une interprétation politico-apologétique des deux traductions (" Bajo el signo de la diplomacia : la reina Cristina en la literatura del Siglo de Oro : del conde Bernardino de Rebolledo, a Calderón y Bances Candano ", in Martínez Ruiz (dir.), España y Suecia en la Época del Barroco (1600-1660), Madrid, Comunidad autónoma de Madrid, 1998, p. 761-762). 
Rostock, qui avait soutenu publiquement une controverse avec le théologien jésuite Godofrido Franken, il compose aussi en 1656 un Discurso apologético où il défend la doctrine du Purgatoire.

La foi militante de Rebolledo ne bride pas sa curiosité intellectuelle, qui brille par son éclectisme. On relève au sein de sa bibliothèque -en plus d'un volume des poésies de Théophile de Viau ${ }^{29}$ et d'une édition des lettres de JeanLouis Guez de $\mathrm{Balzac}^{30}$, tous deux figures du libertinage érudit- un exemplaire du traité De la sagesse $e^{31}$ (1601) de Charron, dont le père Mersenne jugeait avec sévérité la ductilité philosophique : "Il y a plus de difficulté en sa Sagesse, de laquelle on juge diversement : les uns disant qu'elle est séminaire d'irréligion et d'athéisme : les autres confessant que si un homme n'est bien sur ses gardes en la lisant, qu'il court risque d'être ébranlé en sa créance et en sa religion ${ }^{32}$. " De ce que beaucoup tenaient pour un bréviaire de l'impiété, censuré par la Sorbonne et mis à l'Index en 1606, Gassendi avait fait son livre de chevet. "Quel juge plus sensé que Charron ?" écrivait-i ${ }^{33}$. Ainsi cette apologie du doute, «science des sciences [...] pour notre pauvre condition humaine, pleine de ténèbres, faiblesse, incertitude ${ }^{34}$, était-elle parvenue jusqu'à Rebolledo ${ }^{35}$.

"Il n'a rien de plus séant à la nature et à l'esprit humain que la diversité ", écrit Charron ${ }^{36}$. De fait, les liens épistolaires entre Rebolledo et le docteur Juan de Prado attestent l'absence de sectarisme face à un marrane libre-penseur, qui en 1653 , après avoir fui l'Espagne et son Inquisition, s'était converti au judaïsme à Hambourg - centre de rayonnement intellectuel séfarade - avant de bientôt embrasser le déisme aux Pays-Bas ${ }^{37}$. En juillet 1656, Spinoza, avec lequel il avait lié connaissance, était excommunié par la congrégation juive d'Amsterdam et Prado, contraint de se repentir de ses idées hétérodoxes. En juillet 1654, Rebolledo lui adresse un romance où perce une amitié établie ${ }^{38}$,

29. Rebolledo en recommande la lecture ("Tercetos II ", Ocios, p. 159). Sur l'édition des œuvres de Théophile de Viau qu’il pouvait posséder, voir María Concepción Casado Lobato, "La biblioteca de un escritor del siglo XVII : Bernardino de Rebolledo ", Revista de Filología Española, tome LVI, 1973, p. 313-314.

30. Cf. Casado Lobato, art. cit., p. 306.

31. Cf. Casado Lobato, art. cit., p. 310.

32. Marin Mersenne, L'Impiété des déistes, athées et libertins de ce temps, Paris, Pierre Bilaine, 1624, p. 184.

33. Cf. René Pintard, Le Libertinage érudit dans la première moitié du XVIIe siècle, Genève, Éditions Slatkine, 2000 (1943), p. 149.

34. Pierre Charron, De la Sagesse, Rouen, Veuve Durant, 1623, p. 334.

35. L'analyse par Charron du fait religieux devait cependant scandaliser Rebolledo. Confondu avec la coutume, il était présenté comme le " produit des traditions avec ce que cela comporte de mystique, d'arbitraire et d'irrationnel voire d'imposture " (Tullio Gregory, Genèse de la raison classique de Charron à Descartes, Paris, PUF, 2000, p. 124).

36. Ibid., «Préface », p. 16.

37. Il faut toutefois se garder de conclusions hâtives en matière de contacts interconfessionnels où la controverse est absente (Cf. Natalia Muchnik, Une vie marrane. Les pérégrinations de Juan de Prado dans l'Europe du XVIIe siècle, Paris, Honoré Champion, 2005, p. 294-295).

38. Il s'agit du romance LXIII (Ocios, p. 278-282, auquel on associera l'épigramme XLV), qui forme la réponse à un poème que lui avait adressé Prado depuis Hambourg. Rebolledo y 
comme l'indiquent sa liberté de ton et un doux persiflage à l'endroit des pratiques rituelles mosaïques et de ses croyances prophétiques ${ }^{39}$. A aucun moment, Rebolledo ne reproche la volte-face de celui qui a cependant apostasié la foi catholique.

Ni Rebolledo ni Gassendi ne se satisfont d'un scepticisme qui serait liberté de l'indifférence. L'existence de Dieu peut s'appuyer sur la raison ${ }^{40}$. Rebolledo possède ainsi dans sa bibliothèque un exemplaire de La Métaphysique des bons esprits (1642) d'AndréDabillon, lequel, convaincu des vertus de la raison naturelle au service de la foi, se flatte de prouver Dieu à un athée, " plus évidemment que l'on ne prouve les plus évidentes conclusions de la Philosophie " ${ }^{41}$. "Le désir est impérieux, chez ces représentants [de l'apologétique chrétienne], de rendre confiance à la masse des fidèles inquiétée par le rationalisme ou le pyrrhonisme $»^{42}$. Pyrrhonisme justement professé par Charron.

Rebolledo, comme Gassendi, chanoine fidéiste ${ }^{43}$ de Digne, est homme de foi. Pour l'un et l'autre, la liberté philosophique, affranchie des commandements de l'autorité et des empiètements de la théologie, doit pour autant respecter les droits de la religion, les mystères qu'elle dicte, selon la doctrine thomiste de bonne intelligence entre foi et raison. La science consent à des limites qu'il lui est impossible de franchir. Le Syntagma de Gassendi en est la subtile expression : là où la vérité rationnelle est incertaine mais où il existe une vérité révélée ou un dogme proclamé, il appartient à la foi, soit de trancher entre plusieurs vérités philosophiques possibles, soit d'y substituer sa propre vérité.

Rebolledo et Gassendi, cependant, semblent aussi vouloir se réserver la faculté de développer simultanément deux lignes de développement possibles ${ }^{44}$ : la première, scientifique, naturaliste, et la seconde, métaphysique et religieuse. Cet accord, plus ou moins frêle, ressortit à la théorie de la double vérité, soit la coexistence de deux ordres de vérité portant sur les mêmes objets et les mêmes questions, en un dualisme fluent et contingent de la raison physique et de la raison métaphysique. Chez les deux hommes, ce principe relève moins d'un désarroi de l'esprit que d'un programme qu'il faut tenir afin de déjouer un affrontement possible entre science et orthodoxie. Les procédés de la juxtaposition ${ }^{45}$ et de la paralipse forment alors un recours.

exprime son regret de ne pas avoir pu retrouver à Hambourg, en juillet 1654, sa correspondante épistolaire, Christine de Suède, accueillie sous le toit d'Abraham Texeira, membre de la communauté séfarade (cf. Muchnik, op. cit., p. 294). Rebolledo y dépeint la souveraine telle un " no esperado Mesías / en género femenino " (p. 279), en résonance avec le millénarisme des mondes protestant, catholique et séfarade d'alors.

39. Rebolledo ne doute pas que «[...] en sabroso Maná/Se te convierta el rocio, / De que puedas hacer plato / A todos los doce Tribus." (p. 279). Sur l'interprétation millénariste de ces vers, voir Muchnik, op. cit., p. 296.

40. Cf. Sylvie Taussig, "Introduction » de Gassendi, Vie et mours d'Épicure, Paris, Les Belles Lettres, 2006, p. XII.

41. Dabillon, cité par René Pintard, op. cit., p. 70.

42. Pintard, op. cit., p. 71.

43. Cf. Bloch, op. cit., p. 288 sqq., p. 474, p. 481.

44. Cf. Bloch, op. cit., p. 151, p. 162, p. 301, p. 319.

45. Cf. Bloch, op. cit., p. 338 et p. 344-345. 
Les vers que Rebolledo consacre à la querelle de l'héliocentrisme recourent à la même prudence tactique que celle de Gassendi, partisan du copernicanisme : la vérité scientifique, incertaine, laisse possibilité à la foi d'imposer une solution à la raison sans l'imposer directement contre elle.

Rebolledo prône une docte curiosité pour les controverses astronomiques. Quoique les théories autour de la place occupée par la Terre au sein du cosmos soient l'objet de graves polémiques, une curiosité non partisane est salutaire : "Aunque la esfera tan común engaño / Padezca como muchos han creído, / No puede el estudiarla haceros daño ${ }^{46}$. " Le ton est délibérément familier. Avant de succinctement dresser la généalogie de l'héliocentrisme, Rebolledo exprime son impartialité. La liberté philosophique n’entraîne pas au soupçon d'hétérodoxie.

Après la recommandation du traité De Sphaera Mundi (ca. 1230) de Johannes de Sacrobosco ${ }^{47}$-dont la cosmologie ptolémaïque ${ }^{48}$ sera encore étudiée dans de nombreuses universités européennes jusqu'au XVII ${ }^{e}$ siècle-, Rebolledo énumère les principaux jalons antiques de l'héliocentrisme. Héraclide du Pont, en forgeant un modèle où la Terre en rotation sur son axe occupe une position centrale au sein du cosmos, anticipe celui de Tycho-Brahé, qui, dans le dernier quart du XVI ${ }^{\mathrm{e}}$ siècle, conciliait géocentrisme et copernicanisme. Sont cités deux autres partisans de la rotation de la Terre sur son axe : Ecphante de Syracuse et Philolaos de Crotone, qui, le premier, soutint la thèse d'une révolution de notre planète autour d'un feu. Copernic avait reconnu sa dette à leur égard dans une lettre au pape Jules III, en préface à son traité Des Révolutions des orbes célestes (1543). Enfin Rebolledo rappelle que Séleucos de Séleucie fut le seul mathématicien antique à établir la thèse héliocentrique d'Aristarque de Samos :

Heráclites el Póntico y Ecsanto / A la tierra atribuyen movimiento / Sin mudar sitios, en común espanto. / Filolao con mayor atrevimiento / Por la eclíptica juzga que se mueva / Del sol y de la luna en seguimiento. / Seleuco matemático lo aprueba, / Y aun otros más antiguos el camino / Facilitaron a opinión tan nueva ${ }^{49}$.

Rebolledo n'a pas toujours observé avec cette même impartialité les opinions des astronomes favorables à la rotation de la Terre autour du Soleil. La première édition des «Tercetos II », en 1650, affichait une franche hostilité :

De Copérnico huid el desatino, / Que alrededor del sol mueve la tierra : / Contra el sentir humano y aun divino / Galileo Galilei que con el hierra, / Encendió en los modernos la porfia, / Tanto que llega a ser ardiente guerra ${ }^{50}$.

46. «Tercetos II », p. 169

47. Cf. Ibid.

48. Rebolledo mentionne aussi la théorie des épicycles mathématiques, qui fondait la viabilité du géocentrisme ptolémaïque (cf. "Tercetos II ", p. 170). Les calculs et les observations de Copernic et de Kepler la rendront caduque (cf. Alexandre Koyré, La Révolution astronomique : Copernic, Kepler, Borelli, Paris : Herman, 1961).

49. Rebolledo, "Tercetos II », Ocios, Ámberes, Officina Plantiniana, 1650, fo 172v.

50. «Tercetos II », Ocios (1660), p. 169. 
En dix ans, Rebolledo a révisé sa position. L'objurgation a disparu. Le copernicanisme n'est plus un non-sens et Galilée n'est plus un furieux spadassin mais un savant jugeant raisonnablement vraie l'hypothèse de Copernic :

\section{Copérnico a estos tiempos ya vecino / Alrededor del sol traer la quiere, / Contra el sentir} humano y aun divino. / Galilei que le sigue y le prefiere, / Encendió en los modernos la porfia, / Tanto que no hay quien apagarla espere ${ }^{51}$.

Cet infléchissement explique l'ajout dans l'édition de 1660 de ces neuf vers où l'héliocentrisme est rattaché à ses racines antiques.

Rebolledo, à l'instar de Gassendi ${ }^{52}$, juxtapose raison scientifique et vérité de foi, de sorte que celle-ci, sans inhiber la première, impose sa prééminence. Tout se passe comme si, tout en rejetant l'héliocentrisme au nom de la foi, il cherchait à l'épargner, en donnant l'impression de ne parcourir les astronomies antiques et modernes que dans une intention purement historique, comme une hypothèse admissible et en même temps impossible. La contradiction n'est formulée qu'en passant, sans s'y attarder, et même subtilement atténuée par l'emploi du verbe substantivé "sentir » associé à Dieu. La juxtaposition vaut formellement pour irrésolution de la raison.

Après avoir ainsi usé d'une forme de paralipse, Rebolledo choisit le fauxfuyant du bon sens railleur afin d'éteindre la controverse :

Pero yo con Oveno juzgaría / Que acabó de cenar o navegaba / Cuando le pareció que se movia ${ }^{53}$.

Ainsi, par la dérision ${ }^{54}$ adossée aux vérités de foi, flétrit-il la validité de l'héliocentrisme, dont le bon sens établirait aisément les erreurs. Faut-il en prendre acte ? Le trait est un peu gros et l'argument, éculé.

Le copernicanisme militant de Gassendi se développe dans deux traités : l'Institutio Astronomica et le Syntagma, posthume. Dans l'un et l'autre, il répond aux objections du sens commun ou à celles physiques que l'on a coutume d'opposer à l'héliocentrisme. Il y montre patiemment « comment les principes de la mécanique nouvelle (principe d'inertie, principe de relativité, d'indépendance et de composition des mouvements) permettent d'expliquer que le mouvement de la terre n'apparaisse nullement à l'observation vulgaire ${ }^{55}$.

51. Ibid., p. 169-170.

52. Cf. Bloch, op. cit., p. 334.

53. "Tercetos II », p. 170. Le pasteur John Owen jugeait que les calculs des astronomes coperniciens étaient erronés. Rebolledo possédait une édition de ses épigrammes.

54. On rapprochera ce ton de celui du poète Guillaume du Bartas, dont Rebolledo recommande la lecture (cf. «Tercetos II », p. 159), songeant certainement à sa Semaine, ou Création du Monde (1578). Du Bartas, partisan du géocentrisme, en appelait à l'observation simple du monde et du ciel afin de contrecarrer les hypothèses farfelues des astronomes (La Sepmaine (texte de 1581). Ed. Yvonne Bellenger, Paris, STFM, 1981, IV, v. 125 sq.) ; cf. Albert-Marie Schmidt, La poésie scientifique en France au XVIe siècle, Paris, Éditions Rencontre, 1970, p. 321 sqq.

55. Bloch, op. cit., p. 328. 
Rebolledo observe et constate les révolutions du soleil et de la lune mais répugne à admettre celles de la Terre. Partagé entre la raison naturelle vraisemblable et les vérités de la foi ${ }^{56}$, il préfère se réfugier dans la contemplation de l'insondable mystère du monde :

Por la Región diáfana navego / Los Orbes inquiriendo a la Celeste, / [...] / Penetrables sus circulos supone / Contra diversas sectas / El haber visto en parte / Superior al de Marte / Impresiones de fulgidos cometas ; / [...] / Del sol, la luna y los demás planetas / Observo alguna vez los movimientos, / Sin dejarme vencer al de la tierra, / Sentencia de Platón insinuada / Pero no confirmada, / Puesto que tanto a los modernos mueve, / Y de este ángulo breve / Con líneas imperfectas / Por meridianos corro y paralelos / El ámbito espacioso de los cielos, / Sin perdonar constelación ni estrella / Desde la menos clara a la más bella / [...] / Deseando que el alma / De agitación descanse molesta, / Y en nunca de ellas perturbada calma / Con vuelo aspire menos imperfecto / Encubrándose de uno en otro objeto / A la contemplación del soberano, / Último fin de todo afecto humano ${ }^{57}$.

Perplexe quant à la réalité des révolutions terrestres ${ }^{58}$, Rebolledo semble ainsi se soumettre, comme Gassendi ${ }^{59}$, aux objections tirées des Saintes Écritures, lesquelles renferment plus d'érudition et de certitude qu'aucun livre profane ${ }^{60}$. Quoique la vérité de la foi, souveraine et apaisante, suggère un effacement docile de la raison, il est significatif que Rebolledo, en mettant impartialement dos à dos géocentrisme et héliocentrisme, laisse entendre la recevabilité de ce dernier au titre d'hypothèse ${ }^{61}$. Il semble à la fin rejoindre ${ }^{62}$ la solution mitigée de Gassendi : l'inclination, par défaut, pour le système de Tycho Brahé :

Le système de Copernic a beau [...] s'appuyer sur des arguments vraisemblables, il ne peut être démontré de façon irréfutable : [...] au nom d'un agnosticisme ici plus ou moins bien fondé, Gassendi laissera finalement, comme dernier recours pour éviter la théorie de la double vérité, à "ceux qui se font scrupule d'adopter l'hypothèse de Copernic", dans lesquels il parait vouloir se ranger, la possibilité de se rabattre sur le système de Tycho Brahéé .

La contiguïté philosophique entre Rebolledo et Gassendi ne transparaît pas seulement implicitement dans l'œuvre du poète. Elle est discrètement, mais formellement, assumée dans les ultimes lignes d'un discours de réhabilitation de

56. En mars 1616, le traité de Copernic était mis à l'Index par l'Inquisition romaine, et, en 1633, Galilée était condamné à abjurer publiquement sa thèse.

57. "Segunda Selva », p. 495-496.

58. Un roi qui s'y intéresserait perdrait, selon lui, son temps au préjudice des affaires de l'Etat (Selva militar y politica, p. 116v). Cf. «Tercetos II », p. 170.

59. Cf. Bloch, op. cit., p. 328.

60. Cf. ("Tercetos II », p. 170-171).

61. Galilée, dans son Dialogue sur les deux grands systèmes du monde (1632), exposait, lui aussi, de façon impartiale les systèmes aristotélicien et copernicien, en laissant clairement entendre sa préférence pour ce dernier.

62. C'est ce que l'on déduit en effet des vers de la "Segunda Selva " : révolutions du Soleil et de la Lune autour de la Terre immobile, avec pour corrélat - non exprimé - du mouvement orbital d'autres planètes autour du Soleil.

63. Bloch, op. cit., p. 332. 
l'épicurisme, intégré en 1661 à la deuxième édition de la Selva militar y política. Le texte ne révèle pas seulement une lecture minutieuse du De Vita et Moribus Epicuri (1647) où Gassendi rétablissait la grande morale épicurienne : une mention lapidaire de la théorie des espaces imaginaires atteste chez Rebolledo une connaissance de la physique gassendiste ${ }^{64}$ et de la refonte en elle de celle d'Épicure : "También fue de Epicuro aquella opinión tan dificil de refutar de la infinidad de los espacios que él llama reales y los modernos, imaginarios ${ }^{65}$."

La notion ontologique d'espace imaginaire est une des élaborations philosophiques personnelles de Gassendi. L'espace y est décrit comme une réalité infinie, continue, immobile, incorporelle, inaltérable, ni substance ni accident ${ }^{66}$. L'" Espace des Anciens », ressuscité au détriment du "Lieu aristotélicien ${ }^{67}$, va ainsi accompagner l'essor de la physique mécaniste du $\mathrm{XVII}^{\mathrm{e}}$ siècle. L'espace imaginaire gassendiste permet d'expliciter les notions servant de base aux découvertes de Galilée ${ }^{68}$ et de Newton. "L'on peut dire que le langage théologique atteint ici sa limite supérieure : il représente véritablement un outil de la pensée, une armature conceptuelle qui permet un progrès épistémologique ${ }^{69}$."

Parce que l'espace gassendiste est bien un être réel et non un être de raison, il entre en résonance avec celui d'Épicure, comme le rappelle judicieusement Rebolledo. Gassendi donne en effet une interprétation métaphysique à un point de la physique épicurienne ${ }^{70}$. Le matérialisme atomiste est infléchi au profit de la métaphysique et de la théologie chrétienne, laquelle, depuis la fin du MoyenÂge avait développé l'idée d'un espace infini dont seule l'imagination pouvait envisager les dimensions ${ }^{71}$. L'espace gassendiste est incréé, indifférent à tout contenu, et indépendant de l'action divine. Ce cadre vide, infini et réel, rompt avec l'ontologie traditionnelle.

C'est dans sa lettre de réhabilitation d'Épicure que Rebolledo signale à mots voilés sa familiarité vis-à-vis de l'œuvre de Gassendi :

En cuatro tomos que dicen que ha dado a luz en Francia Gasendo de su [cf. d'Épicure] vida y filosofía física y moral, hallará quien las deseare muchas más noticias : que a mi aun

64. Physique qui, chez Gassendi, est inséparable de la Métaphysique (cf. Bloch, op. cit., p. 172).

65. «Este papel escribió el autor al Señor barón Don Juan de Goes ", Selva militar y política, Madrid, Antonio de Sancha, 1661, t. II, p. 495.

66. Cf. Gassendi, Syntagma, Livre « De Loco et Tempore », I, 179a. Cf. Bloch, op. cit., p. 174175 , p. 188 sqq.

67. Cf. Bloch, op. cit., p. 172, p. 200-201 et Karl Schuhmann, «Le Vocabulaire de l'espace», in Yves Charles Zarka (ed.), Hobbes et son vocabulaire, Paris, Vrin, 1992, p. 69 sqq.

68. Cf. Bloch, op. cit., p. 194.

69. Bloch, op. cit., p. 317.

70. Cf. Bloch, op. cit., p. 301, p. 317.

71. Pour une généalogie de l'idée d'espace imaginaire, voir Schuhmann, art. cit., p. 67. L'importance de théologiens espagnols, tels Francisco Toledo, y est soulignée. 
éstas me parecen demasiadas, y aun a él se lo deben de parecer también, pues cuanto en su favor se dice no templa nada de lo que padece ${ }^{72}$.

Ce n'est pas là un simple renvoi à la biographie d'Épicure mais une clef offerte au lecteur averti.

On ne procédera pas ici à l'examen de l'épicurisme stoïcisé de Rebolledo, qui en rétablit la dignité édifiante, mais à l'établissement, au moyen de quelques preuves formelles, du filigrane du De Vita et Moribus Epicuri. A trop souligner dans ce texte une contiguiité philosophique - certes indéniable - avec la Defensa de Epicuro par Quevedo, on a effacé en effet la trace de ce texte nourricier.

Puisant aux mêmes sources (Diogène Laërce, Plutarque, Sénèque, Cicéron, Lucrèce, Juvénal, Lactance, Sextus Empiricus), Quevedo, Gassendi et Rebolledo convoquent souvent les mêmes citations. Certaines, divergentes, suggèrent cependant que Rebolledo fit du traité de Gassendi son véritable ferment. Ainsi celles tirées du De Rerum Natura, via le De Vita, où Lucrèce chante la géniale acuité d'Épicure, comparé à un Dieu, pour avoir percé les secrets de la nature ${ }^{73}$. Rebolledo reproduit aussi les citations de Juvénal ${ }^{74}$ retenues par Gassendi, et certaines de Cicéron tirées du traité Des vrais biens et des vrais maux, où l'épicurisme doit être distingué de sa version dégradée par la faute de fâcheux disciples ${ }^{75}$. Enfin, l'apologie de la frugalité d'Épicure et sa morale de la continence heureuse, étrangère à toute "volupté perverse ", s'appuient sur un répertoire de citations d'Alexandre d'Alexandrie, Celius Rhodiginus, Raffaello Maffei dit Volaterranus, Pic de la Mirandole, François Philelphe et saint Grégoire de Nazianze, toutes empruntées à Gassendi sans exception ${ }^{76}$.

La poésie scientifique de Rebolledo et sa lettre de réhabilitation d'Épicure attestent ainsi l'influence du gassendisme et la fécondité de ses compromis philosophiques auprès de penseurs chrétiens antidogmatiques et sceptiques, soucieux d'une bonne intelligence entre foi et raison, comme au siècle suivant, Benito Feijoo, en qui perdure ce modèle.

72. «Este papel... », p. 495-496.

73. Ibid., p. 484 ; Gassendi, Vie et mours, II, 4, \$4, p. 127, p. 129 ; IV, 2, \$ 2, p. 9-11.

74. Ibid., p. 484 ; Gassendi, ibid., VI, 3, \$1-2, p. 101.

75. Ibid., p. 487 ; Gassendi, ibid., IV, 8, \$2, p. 41 ; VI, 5, \$1, p. 113 ; VII, 8, \$1, p. 185.

76. Ibid., p. 492, p. 494 ; Gassendi, ibid., VII, 7, \$ 5, p. 177-179. 
